Bull. Soc. math. France

131 (1), 2003, p. 1-22

\title{
SOLUTIONS GLOBALES DE \\ L'ÉQUATION DES ONDES SEMI-LINÉAIRE CRITIQUE À COEFFICIENTS VARIABLES
}

\author{
Par Slim Ibrahim \& Mohamed Majdoub
}

RÉSumÉ. - Dans ce travail, on s'intéresse à l'existence globale de solutions classiques et au sens de Shatah-Struwe de l'équation des ondes critique à coefficients variables en dimension $d$ d'espace

$$
\square_{A} u+|u|^{4 /(d-2)} u=\partial_{t}^{2} u-\operatorname{div}\left(A(x) \cdot \nabla_{x} u\right)+|u|^{4 /(d-2)} u=0, \quad \mathbb{R}_{t} \times \mathbb{R}_{x}^{d},
$$

où $A$ est une fonction régulière à valeurs dans les matrices $d \times d$ définies positives, valant l'identité en dehors d'un compact fixe.

ABStRACT (Global solutions for the critical nonlinear wave equation in variable coefficients)

In this work, we study the existence of both global smooth and Shatah-Struwe's solutions of the critical wave equation in variable coefficients in dimension $d$ of space

$$
\square_{A} u+|u|^{4 /(d-2)} u=\partial_{t}^{2} u-\operatorname{div}\left(A(x) \cdot \nabla_{x} u\right)+|u|^{4 /(d-2)} u=0, \quad \mathbb{R}_{t} \times \mathbb{R}_{x}^{d},
$$

where $A$ is a regular function valued in the space of $d \times d$ positive definite matrix and which is the identity outside a fixed compact.

Texte reçu le 4 avril 2001, révisé le 21 décembre 2001, accepté le 7 février 2002

Slim Ibrahim, Département de Mathématiques, Faculté des Sciences de Bizerte, Jarzouna 7021-Bizerte (Tunisie) • E-mail : slim.ibrahim@fsb.rnu.tn

Mohamed Majdoub, Département de Mathématiques, Faculté des Sciences de Tunis, Campus Universitaire 1060-Tunis (Tunisie) - E-mail : mohamed.majdoub@fst.rnu.tn Classification mathématique par sujets (2000). - 35-XX, 35Lxx, 35L05.

Mots clefs. - Équation des ondes, inégalités de Strichartz, existence globale, cônes géodésiques. 


\section{Introduction}

L'objectif de ce travail est d'établir des résultats d'existence et d'unicité de solutions du problème de Cauchy associé à l'équation des ondes semi-linéaire critique à coefficients variables en dimension $d \geq 3$

$$
\begin{array}{r}
\square_{A} u+|u|^{p_{c}-1} u:=\partial_{t}^{2} u-\operatorname{div}\left(A(x) \nabla_{x} u\right)+|u|^{p_{c}-1} u=0 \\
\text { sur } \mathbb{R}_{t} \times \mathbb{R}_{x}^{d},
\end{array}
$$

où $p_{c}=(d+2) /(d-2), A$ est une fonction $\mathcal{C}^{\infty}$ à valeurs dans les matrices $d \times d$ symétriques et vérifiant l'hypothèse

$$
\left\{\begin{array}{l}
c_{0}|\xi|^{2} \leq A(x) \xi \cdot \xi \leq c_{0}^{-1}|\xi|^{2}, \quad \forall \xi \in \mathbb{R}^{d}, \\
A(x) \equiv \mathrm{Id}, \quad \forall x \in \mathbb{R}^{d},|x| \geq R_{0},
\end{array}\right.
$$

pour des constantes $R_{0}>0$ et $0<c_{0} \leq 1$ données. L'opérateur $\operatorname{div}\left(A(x) \nabla_{x} \cdot\right)$ représente alors une perturbation locale en espace de l'opérateur Laplacien $\Delta_{x}$. Lorsque $A(x) \equiv \mathrm{Id}$, cas constant, nous retrouvons l'opérateur d'Alembertien

$$
\square=\partial_{t}^{2}-\Delta_{x} \quad \text { sur } \quad \mathbb{R}_{t} \times \mathbb{R}_{x}^{d} .
$$

L'équation (1) est un cas particulier d'une classe plus générale d'équations du type

$$
\square_{A} u+|u|^{p-1} u=0,
$$

où $p$ est un réel dans $] 1,+\infty[$.

La question d'existence globale de solutions pour le problème de Cauchy associé à l'équation (2) est fondamentale. Elle a fait l'objet de nombreux travaux depuis les années soixante. Rappelons d'abord les résultats dans le cas constant.

- Pour $1<p<p_{c}$, cas sous-critique, J. Ginibre et G. Velo [4] ont démontré que pour des données

$$
\left(u(0), \partial_{t} u(0)\right) \in\left(\dot{H}^{1}\left(\mathbb{R}^{d}\right) \cap L^{p+1}\left(\mathbb{R}^{d}\right)\right) \times L^{2}\left(\mathbb{R}^{d}\right),
$$

il existe une unique $u$,

$$
u \in \mathcal{C}\left(\mathbb{R}, \dot{H}^{1}\left(\mathbb{R}^{d}\right) \cap L^{p+1}\left(\mathbb{R}^{d}\right)\right), \quad \partial_{t} u \in \mathcal{C}\left(\mathbb{R}, L^{2}\left(\mathbb{R}^{d}\right)\right),
$$

solution forte du problème de Cauchy associé à l'équation (2).

- Pour $p=p_{c}$, cas critique, ce problème a d'abord été résolu dans le cas radial par M. Struwe [18], puis dans le cas général par M. Grillakis [6], [7] pour les dimensions $d$ telles que $3 \leq d \leq 5$ et récemment J. Shatah-M. Struwe [15], [16] l'ont prouvé pour les autres dimensions. Précisément, ils ont prouvé l'existence globale et l'unicité de solutions dans la classe dite de Shatah-Struwe

$$
u \in \mathcal{C}\left(\mathbb{R}, \dot{H}^{1}\left(\mathbb{R}^{d}\right)\right) \cap L_{\text {loc }}^{p_{c}}\left(\mathbb{R}, L^{2 p_{c}}\left(\mathbb{R}^{d}\right)\right), \quad \partial_{t} u \in \mathcal{C}\left(\mathbb{R}, L^{2}\left(\mathbb{R}^{d}\right)\right) .
$$

La condition inhabituelle, $u \in L_{\text {loc }}^{p_{c}}\left(\mathbb{R}, L^{2 p_{c}}\left(\mathbb{R}^{d}\right)\right)$, permet de considérer le terme $|u|^{p_{c}-1} u$ dans $(1)$ comme un terme source dans $L_{\text {loc }}^{1}\left(\mathbb{R}, L^{2}\left(\mathbb{R}^{d}\right)\right)$ et d'obtenir des estimations d'énergie.

TOME $131-2003-\mathrm{N}^{\mathrm{O}} 1$ 
La question d'unicité de solutions dans $\mathcal{C}\left(\mathbb{R}, \dot{H}^{1}\left(\mathbb{R}^{d}\right)\right) \cap \mathcal{C}^{1}\left(\mathbb{R}, L^{2}\left(\mathbb{R}^{d}\right)\right)$ et non pas dans $L_{\text {loc }}^{p_{c}}\left(\mathbb{R}, L^{2 p_{c}}\left(\mathbb{R}^{d}\right)\right)$ est encore ouverte. Cependant H. Bahouri et P. Gérard [1] ont montré la stabilité des solutions de Shatah-Struwe pour la convergence faible dans l'espace d'énergie.

- Pour $p>p_{c}$, cas sur-critique, le problème d'existence et d'unicité de solutions fortes est ouvert. Cependant dans un travail récent, G. Lebeau [13] a obtenu un résultat d'instabilité pour les solutions radiales et à valeurs réelles d'une équation des ondes sur-critique dans $\mathbb{R}^{3}$. Pour une bibliographie détaillée, voir C. Zuily [19].

Dans le cas variable, L.V. Kapitanski [11], [10] a établi l'existence globale de solutions fortes pour les puissances sous-critiques.

Dans ce travail nous nous intéressons au cas où $p=p_{c}$. Signalons d'abord que dans [12], Kapitanski a obtenu des résultats d'existence et d'unicité de solutions faibles. Notre premier résultat concerne l'existence globale de solutions régulières. Précisément, nous montrons les résultats énoncés dans [8].

THÉORÈme 1.1. - On suppose que $3 \leq d<6$, et que la fonction $A(x)$ vérifie l'hypothèse $(\mathcal{H})$. Alors si $s>\frac{1}{2} d+2$ et $\left(u_{0}, u_{1}\right) \in H^{s}\left(\mathbb{R}^{d}\right) \times H^{s-1}\left(\mathbb{R}^{d}\right)$, le problème

$$
\square_{A} u+|u|^{p_{c}-1} u=0, \quad u(0, .)=u_{0}, \quad \partial_{t} u(0, .)=u_{1},
$$

admet une unique solution globale $u \in C^{2}\left(\mathbb{R} \times \mathbb{R}^{d}\right)$.

REMARQUe 1.1. - 1) La restriction sur la dimension est dûe à l'utilisation de l'injection de Sobolev $W^{m, 2 d /(d-2)}\left(\mathbb{R}^{d}\right) \hookrightarrow L^{\infty}\left(\mathbb{R}^{d}\right)$ pour $m>\frac{1}{2} d-1$.

2) La preuve du théorème 1.1 est basée sur un résultat de base d'existence locale et d'explosion (voir lemme 3.3).

La démonstration du théorème 1.1 se fait par l'absurde, en montrant des estimations $L^{\infty}$ sur la solution maximale $u$. Pour ce faire, nous adoptons malgré sa rigidité, la méthode de J.Shatah-M. Struwe [15]. Cette méthode s'appuie sur un résultat clé, exprimant la non concentration de la partie non linéaire de l'énergie (et par suite de l'énergie). L'idée consiste à exhiber des hypersurfaces de $\mathbb{R}_{t} \times \mathbb{R}_{x}^{d}$ dépendant de la géométrie de l'opérateur $\square_{A}$ et qui jouent, dans le cas constant, le même rôle que les cônes d'ondes usuels. Ce résultat sera souvent utilisé avec les estimations a priori suivantes, vérifiées par la solution du problème linéaire.

Soit $T>0, s \in \mathbb{R}$ et notons par $v$ la solution de

$$
\left\{\begin{array}{l}
\partial_{t}^{2} v+G(t) v=f(t) \quad \text { sur }[0, T] \times \mathbb{R}^{d}, \\
v(0)=v_{0}, \quad \partial_{t} v(0)=v_{1},
\end{array}\right.
$$

où $f(t) \in L^{1}\left([0, T] ; H^{s}\left(\mathbb{R}^{d}\right)\right)$ et $G(t)$ est un opérateur pseudo-différentiel classique d'ordre 2 dépendant d'une manière $\mathcal{C}^{\infty}$ en $t$, ayant un symbole principal $G_{2}$ vérifiant la condition d'uniforme ellipticité $G_{2}(t, x, \xi) \geq c|\xi|^{2}$, et tel que 
tous les termes $G_{2-j}, j=0,1, \ldots$, du développement asymptotique du symbole principal sont indépendants de $x$ en dehors d'une boule fixe de $\mathbb{R}^{d}$. Alors nous avons :

Lemme 1.1 (estimation de l'énergie). - Soit $\left(v_{0}, v_{1}\right) \in H^{s+1} \times H^{s}$. Le problème (4) admet une unique solution $v$ satisfaisant

$$
\begin{aligned}
& \sup _{t \in[0, T]}\left(\left\|\partial_{t} v(t)\right\|_{H^{s}}+\|\nabla v(t)\|_{H^{s}}\right) \\
& \quad \leq c(s)\left(\left\|v_{1}\right\|_{H^{s}}+\left\|\nabla v_{0}\right\|_{H^{s}}+\int_{0}^{T}\|f(t)\|_{H^{s}} \mathrm{~d} t\right),
\end{aligned}
$$

où la constante $c(s)>0$ est indépendante de $v_{0}, v_{1}, f$.

Lemme 1.2 (inégalités de Strichartz). - Soit $\left(v_{0}, v_{1}\right) \in \dot{H}^{1} \times L^{2}$. Pour tout couple de Strichartz $(q, r)$, i.e

$$
\frac{1}{q}+\frac{d}{r}=\frac{d}{2}-1, \quad q \geq \frac{d+1}{d-1} \quad \text { et } \quad q>2 \quad \text { si } d=3,
$$

la solution $v$ du problème (4) vérifie

$$
\begin{aligned}
& \|v\|_{L^{q}\left([0, T], L^{r}\left(\mathbb{R}^{d}\right)\right)} \\
& \quad \leq c_{q}\left(\left\|v_{1}\right\|_{L^{2}\left(\mathbb{R}^{d}\right)}+\left\|\nabla v_{0}\right\|_{L^{2}\left(\mathbb{R}^{d}\right)}+\int_{0}^{T}\|f(t)\|_{L^{2}\left(\mathbb{R}^{d}\right)} \mathrm{d} t\right),
\end{aligned}
$$

avec une constante universelle $c_{q}$.

Les inégalités de Strichartz ont été établies par Ginibre-Velo [5] dans le cas constant et Kapitanski [9] dans le cas où la métrique $G$ satisfait les hypothèses décrites ci-dessus. Signalons enfin que dans le cas de coefficients peu réguliers, H. Smith a démontré dans un travail récent (voir [17]) les estimations de Strichartz pour des opérateurs à coefficients seulement $\mathcal{C}^{1,1}$.

Ces estimations seront souvents couplées avec un lemme d'absorption que nous rappelons.

LEmme 1.3 (lemme de bootstrap). - Soit $X:[0, T] \longrightarrow \mathbb{R}_{+}$une fonction continue telle que

$$
X(t) \leq a+b X(t)^{\theta},
$$

avec $a, b>0, \theta>1$,

$$
a<\left(1-\frac{1}{\theta}\right) \frac{1}{(\theta b)^{1 / \theta}} \quad \text { et } \quad X(0) \leq \frac{1}{(\theta b)^{1 /(\theta-1)}} .
$$

Alors, pour tout $t \in[0, T]$, on a

$$
X(t) \leq \frac{\theta}{\theta-1} a .
$$

Dans notre second résultat, nous nous intéressons aux solutions fortes au sens de Shatah-Struwe. Précisément, nous montrons le

TOME $131-2003-\mathrm{N}^{\mathrm{O}} 1$ 
ThÉORÈme 1.2. - Soient $d \geq 3$ et $\left(u_{0}, u_{1}\right) \in \dot{H}^{1}\left(\mathbb{R}^{d}\right) \times L^{2}\left(\mathbb{R}^{d}\right)$. L'équation (1) admet une unique solution

$$
u \in \mathcal{C}\left(\mathbb{R}, \dot{H}^{1}\left(\mathbb{R}^{d}\right) \cap L_{\text {loc }}^{p_{c}}\left(\mathbb{R}, L^{2 p_{c}}\left(\mathbb{R}^{d}\right)\right) ; \quad \partial_{t} u \in \mathcal{C}\left(\mathbb{R}, L^{2}\left(\mathbb{R}^{d}\right)\right)\right.
$$

vérifiant $u(0,)=.u_{0}$ et $\partial_{t} u(0,)=.u_{1}$.

La preuve de ce théorème se fait en plusieurs étapes. D'abord, nous montrons par une méthode de point fixe, l'existence locale d'une solution au sens de Shatah-Struwe. Puis, nous prouvons l'unicité de telles solutions comme dans [16]. Enfin, en utilisant le lemme fondamental (lemme 3.2), nous prolongeons la solution locale.

Indiquons maintenant le plan de cet article. Dans le prochain paragraphe, nous rappelons les notions géométriques nécessaires à l'introduction des cônes géodésiques. Le paragraphe 3 est consacré à la preuve du théorème 1.1 : d'abord, un calcul précis sur ces cônes permet d'établir un lemme fondamental exprimant la non concentration de l'énergie locale. Ensuite, nous utilisons les inégalités de Strichartz pour obtenir des estimations $L^{\infty}$ de la solution maximale du problème (3). Enfin, un résultat d'existence locale et d'explosion permet de conclure. Au dernier paragraphe, par une méthode du point fixe nous prouvons l'existence locale de solutions fortes du problème (3). L'unicité et l'existence globale sont obtenues via les inégalités de Strichartz. En appendice nous établissons une version localisée des estimations de Strichartz dans les cônes géodésiques. La preuve est basée sur une idée de Bahouri-Gérard.

Remerciements. - Les auteurs tiennent à remercier le professeur Hajer Bahouri d'avoir proposé cette question. Ils expriment aussi leurs remerciements au professeur Patrick Gérard pour ses remarques et ses suggestions. Ils remercient enfin le rapporteur anonyme pour sa contribution à l'élaboration de cette version finale de l'article.

\section{Cônes géodésiques}

Notons $a_{i j}(x)$ les coefficients de la matrice $A(x)$ et $a^{i j}(x)$ ceux de $A^{-1}(x)$. Nous munissons $\mathbb{R}^{d}$ de la métrique Riemannienne $g$ définie à partir de la matrice $A^{-1}(x)$ par

$$
g=\sum_{i j} a^{i j} \mathrm{~d} x^{i} \otimes \mathrm{d} x^{j} .
$$

Pour tout point $x \in \mathbb{R}^{d}$, et tous vecteurs $u, v \in T_{x} \mathbb{R}^{d}$, nous noterons par $g_{x}(u, v)$ le produit scalaire $\left\langle A^{-1}(x) u, v\right\rangle$ et par $\|\cdot\|_{x}$ la norme correspondante. Nous rappelons que la longueur d'une courbe $\gamma:[a, b] \longrightarrow \mathbb{R}^{d}, \mathcal{C}^{1}$ par morceaux, est

$$
L(\gamma)=\int_{[a, b]}\left\|\gamma^{\prime}(t)\right\|_{\gamma(t)} \mathrm{d} t
$$

BULletin DE LA SOCIÉtÉ MATHÉMATIQUE DE FRANCE 
La distance géodésique de deux points $x$ et $y$, notée $d_{g}(x, y)$, est donnée par l'infimum des longueurs des courbes joignant ces deux points.

2.1. Application exponentielle. - On rappelle que les géodésiques de $\left(\mathbb{R}^{d}, g\right)$ sont les courbes qui, en coordonnées locales, sont régies par le système d'équations

$$
\begin{aligned}
\ddot{x}^{k}(t)+\frac{1}{2} \dot{x}^{i} \dot{x}^{j} g^{k \ell}(x(t))\left(2 \partial_{i} g_{j \ell}(x(t))-\partial_{\ell} g_{i j}(x(t))\right) & =0, \\
k & =1,2, \ldots, d .
\end{aligned}
$$

Pour $x \in \mathbb{R}^{d}$ et $v \in T_{x} \mathbb{R}^{d}$, notons par $C_{v}$ la géodésique vérifiant $C_{v}(0)=x$ et $\dot{C}_{v}(0)=v$. L'application exponentielle en $x$ est

$$
\exp _{x}: T_{x} \mathbb{R}^{d} \longrightarrow \mathbb{R}^{d}, \quad v \longmapsto C_{v}(1) .
$$

Cette application n'est pas a priori bien définie. Mais vu l'homogéneité de (7), nous avons (voir par exemple [3]) :

Lemme 2.1. - Pour tout $x \in \mathbb{R}^{d}$, il existe $\varepsilon>0$ et $U_{x}$ un voisinage de $x$ dans $\mathbb{R}^{d}$ tels que l'application

$$
\exp _{x}: V_{x}=\left\{w,\|w\|_{x}<\varepsilon\right\} \longrightarrow U_{x}
$$

soit un $\mathcal{C}^{\infty}$ difféomorphisme et

$$
\forall y \in U_{x}, \quad d_{g}(x, y)=\left\|\exp _{x}^{-1}(y)\right\|_{x} .
$$

Cette application vérifie les propriétés décrites dans le résultat suivant connu sous le nom du lemme de Gauss.

Lemme 2.2 (lemme de Gauss). - Soit $x \in \mathbb{R}^{d}$ et $V_{x}$ le voisinage donné par le lemme 2.1. Nous avons alors :

$$
\begin{gathered}
D \exp _{x}(0) v=v, \quad \text { pour tout } v \in T_{x} \mathbb{R}^{d}, \\
\left\|D \exp _{x}(v) \cdot v\right\|_{\exp _{x}(v)}=\|v\|_{x}, \quad \text { pour tout } v \in V_{x}, \\
\text { si } g_{x}(v, w)=0 \text { alors } g_{\exp _{x}(v)}\left(D \exp _{x}(v) v, D \exp _{x}(v) w\right)=0 .
\end{gathered}
$$

On renvoie à [3], prop. 2.93, pour les détails et les démonstration des résultats ci-dessus.

Les identités (10) et (11) du lemme 2.2 traduisent le fait que $D \exp _{x}(v)$ est une isométrie dans la direction de $v$.

En tout point $x_{0} \in \mathbb{R}^{d}$, nous définissons l'application $\varphi_{x_{0}}$ par

$$
\varphi_{x_{0}}(x):=d_{g}\left(x, x_{0}\right) .
$$

Nous avons alors le résultat suivant.

TOME $131-2003-\mathrm{N}^{\mathrm{O}} 1$ 
Proposition 2.1. - Soit $x_{0} \in \mathbb{R}^{d}$. Il existe un voisinage $U_{x_{0}}$ tel que l'application $\varphi_{x_{0}} \in \mathcal{C}^{\infty}\left(U_{x_{0}} \backslash\left\{x_{0}\right\}, \mathbb{R}_{+}^{*}\right)$ et

$$
\begin{aligned}
& A(x) \nabla \varphi_{x_{0}} \cdot \nabla \varphi_{x_{0}}=1 \text { sur } U_{x_{0}} \backslash\left\{x_{0}\right\}, \\
& \operatorname{div}\left(\varphi_{x_{0}} A(x) \nabla \varphi_{x_{0}}\right)=d+O\left(\varphi_{x_{0}}\right), \\
& B(x):=A(x) \nabla\left(\varphi_{x_{0}}^{t} \nabla \varphi_{x_{0}}\right)-\operatorname{Id}=O\left(\varphi_{x_{0}}\right), \\
& c\left(x_{0}\right)\left|x-x_{0}\right| \leq \varphi_{x_{0}}(x) \leq c^{-1}\left(x_{0}\right)\left|x-x_{0}\right|,
\end{aligned}
$$

où $c\left(x_{0}\right)$ est une constante positive.

Démonstration de la proposition 2.1. - Nous supposons que $x_{0}=0$, et nous prenons $U_{0}$ le voisinage de 0 donné par le lemme 2.1 .

Comme $\varphi_{0}(x)=\left\|\exp _{0}^{-1}(x)\right\|_{0}$ pour tout $x \in U_{0}$, alors $\varphi_{0} \in \mathcal{C}^{\infty}\left(U_{0}\left\{\{0\}, \mathbb{R}_{+}^{*}\right)\right.$. Soit $v \in V_{0}$, alors pour tout $t>0$, assez proche de 1 , nous avons en vertu de (8)

$$
\varphi_{0}\left(\exp _{0}(t v)\right)=t\|v\|_{0} .
$$

En différentiant (16) par rapport à $t$, et en prenant $t=1$, nous avons

$$
D \varphi_{0}\left(\exp _{0}(v)\right) D \exp _{0}(v) \cdot v=\|v\|_{0} .
$$

Par ailleurs, pour tout $v \in V_{0}$ nous avons $\varphi_{0}\left(\exp _{0}(v)\right)=\|v\|_{0}$. En différentiant l'égalité précédente par rapport à $v$ et en l'appliquant à $w \in v^{\perp}$ (i.e. $\left.g_{0}(w, v)=0\right)$, on obtient

$$
\left\langle D \varphi_{0}\left(\exp _{0}(v)\right) D \exp _{0}(v), w\right\rangle=0
$$

Si nous notons par $\widetilde{\nabla}$ le gradient défini sur $\left(\mathbb{R}^{d}, A^{-1}(x)\right)$, les expressions $(17)$ et (18) sont équivalentes à

$$
\begin{gathered}
g_{x}\left(\widetilde{\nabla} \varphi_{0}\left(\exp _{0}(v)\right), D \exp _{0}(v) \cdot v\right)=\|v\|_{0}, \\
g_{x}\left(\widetilde{\nabla} \varphi_{0}\left(\exp _{0}(v)\right), D \exp _{0}(v) \cdot w\right)=0
\end{gathered}
$$

D'après (10) et (11) du lemme 2.2, nous avons

$$
\widetilde{\nabla} \varphi_{0}\left(\exp _{0}(v)\right)=\frac{D \exp _{0}(v) \cdot v}{\|v\|_{0}} \text { et } \| \tilde{\nabla} \varphi_{0}\left(\exp _{0}(v) \|_{\exp _{0}(v)}=1\right.
$$

Comme $\widetilde{\nabla}=A(x) \nabla$, l'assertion (12) est alors démontrée.

Pour prouver (13), remarquons d'abord que $\varphi_{0}^{2}$ est $\mathcal{C}^{\infty}$ au voisinage de 0 d'après (16). Il suffit donc, compte tenu de la formule de Taylor, de calculer la différentielle en 0 de la fonction régulière $A \nabla\left(\varphi_{0}^{2}\right)$. D'après (21), nous savons que la composée de cette fonction avec l'exponentielle est $D \exp _{0}(v) \cdot v$, dont la différentielle en $v=0$ n'est autre que l'identité. Par composition des différentielles, la différentielle de $A \nabla\left(\varphi_{0}^{2}\right)$ en 0 est donc l'identité. En prenant la trace, on obtient la divergence. Nous concluons en utilisant (15). 
Pour la preuve de (14), nous écrivons

$$
A \nabla\left(\varphi_{0}^{t} \nabla \varphi_{0}\right)-\mathrm{Id}=A \nabla\left[^{t}\left(\varphi_{0} A \nabla \varphi_{0}\right) A^{-1}\right]-\mathrm{Id} .
$$

Or $\left(\varphi_{0} A \nabla \varphi_{0}\right)(x)=D \exp _{0}(v) \cdot v$ avec $x=\exp _{0}(v)$, ce qui entraîne que

$$
A \nabla\left[\left(\varphi_{0} A \nabla \varphi_{0}\right)\right] A^{-1}(0)=\mathrm{Id} .
$$

Un raisonnement analogue à celui fait dans la preuve de (13) donne le résultat. Enfin, en écrivant près de 0

$$
\varphi_{0}^{2}(x)=\frac{1}{2} D^{2}\left(\varphi_{0}^{2}\right)(0)(x, x)+o\left(|x|^{2}\right),
$$

nous obtenons (15).

REMARQUe 2.1. - 1) Dans le cas constant, pour tout $x_{0} \in \mathbb{R}^{d}$, nous avons $U_{x_{0}}=\mathbb{R}^{d}$ et $\varphi_{x_{0}}(x)=\left|x-x_{0}\right|$.

2) Nous constatons que les géodésiques de la variété riemannienne $\left(\mathbb{R}^{d}, A^{-1}(x)\right)$ sont en fait les rayons (projection en $\left.(t, x)\right)$ des bicaractéristiques nulles du champ hamiltonien associé au symbole principal de $\square_{A}$ définies par les solutions de

$$
\begin{gathered}
\dot{x}(t)=2 A(x) \xi, \quad \dot{\xi}=-\nabla_{x}\left(a_{i j} \xi^{i} \xi^{j}\right), \quad \dot{t}=-2 \tau, \dot{\tau}=0, \\
t(0)=t_{0}, x(0)=x_{0}, \quad \tau(0)=\tau_{0}, \quad \xi(0)=\xi_{0},
\end{gathered}
$$

issues des points $\left(t_{0}, x_{0}, \tau_{0}, \xi_{0}\right)$ tel que $A\left(x_{0}\right) \xi_{0} \cdot \xi_{0}=\tau_{0}^{2}$ de l'espace cotangent $T^{*}\left(\mathbb{R}_{t} \times \mathbb{R}_{x}^{d}\right)$. En effet, toute géodésique $(-2 t, x(-2 t))$ est un rayon de la bicaractéristique nulle $\Gamma=\left(-2 t, x(-2 t), 1, \frac{1}{2} A^{-1}(x(t)) \dot{x}(t)\right)$ et réciproquement. Les solutions de $(7)$ sont globales ce qui fait de $\mathbb{R}^{d}$ une variété riemannienne complète.

3) L'hypersurface de $\mathbb{R}^{d}$ définie par $\left\{(t, x) / t^{2}=\varphi_{x_{0}}^{2}(x)\right\}$ est tissée de géodésiques. Pour tout point $z_{0}=\left(t_{0}, x_{0}\right) \in\left[0,+\infty\left[\times \mathbb{R}^{d}\right.\right.$, nous appelons cône géodésique issu de $z_{0}$ l'ensemble des points $(t, x)$ tels que $\varphi_{x_{0}}^{2}(x) \leq\left(t-t_{0}\right)^{2}$ et $x \in$ $U_{x_{0}}$.

Introduisons maintenant les notations suivantes.

2.2. Notations. - Pour $Q \subset \mathbb{R} \times \mathbb{R}^{d}$ et $S<T$, nous notons par

$$
Q_{S}^{T}:=\{z=(t, x) \in Q \text { tel que } S \leq t \leq T\},
$$

la partie tronquée de $Q$ entre les instants $S$ et $T$, et

$$
\|u\|_{L^{q}\left(L^{r}(Q)\right)}^{q}:=\int_{\pi_{t}(Q)}\left(\int_{Q_{t}}|u(t, x)|^{r} \mathrm{~d} x\right)^{q / r} \mathrm{~d} t
$$

où $Q_{t}$ désigne la trace de $Q$ à l'instant t; $Q_{t}=\{x,(t, x) \in Q\}$ et $\pi_{t}$ la première projection; $\pi_{t}: \mathbb{R}_{t} \times \mathbb{R}_{x}^{d} \rightarrow \mathbb{R}_{t}$.

TOME $131-2003-\mathrm{N}^{\mathrm{O}} 1$ 
Pour tout $z_{0}=\left(t_{0}, x_{0}\right) \in \mathbb{R} \times \mathbb{R}^{d}$, nous définissons

$$
K\left(z_{0}\right):=\left\{z=(t, x) \in \mathbb{R} \times U_{x_{0}} \text { tel que } \varphi_{x_{0}}(x) \leq t_{0}-t\right\}
$$

le cône rétrograde de sommet $z_{0}$, et pour $t$ donné

$$
D\left(t, z_{0}\right):=\left\{x \in U_{x_{0}} \text { tel que } \varphi_{x_{0}}(x) \leq t_{0}-t\right\}
$$

sa section à l'instant $t$. Le manteau du cône $K\left(z_{0}\right)$, noté $M\left(z_{0}\right)$, est défini par

$$
M\left(z_{0}\right):=\left\{z=(t, x) \in \mathbb{R} \times U_{x_{0}} \text { tel que } \varphi_{x_{0}}(x)=t_{0}-t\right\} .
$$

Nous définissons aussi le cône d'avenir $\widetilde{K}\left(z_{0}\right)$ par

$$
\widetilde{K}\left(z_{0}\right):=\left\{z=(t, x) \in \mathbb{R} \times U_{x_{0}} \text { tel que } \varphi_{x_{0}}(x) \leq t-t_{0}\right\} .
$$

Pour toute fonction $u=u(t, x)$, nous définissons la densité d'énergie, l'énergie locale, la densité de flux et le flux de $u$ respectivement par

$$
\begin{aligned}
e(u) & :=\frac{1}{2}\left(\left(\partial_{t} u\right)^{2}+\left|A^{\frac{1}{2}} \nabla_{x} u\right|^{2}\right)+\frac{1}{p_{c}+1}|u|^{p_{c}+1}, \\
E\left(u, D\left(t, z_{0}\right)\right) & :=\int_{D\left(t, z_{0}\right)} e(u) \mathrm{d} x=E_{0}\left(u, D\left(t, z_{0}\right)\right)+\int_{D\left(t, z_{0}\right)} \frac{|u|^{p_{c}+1}}{p_{c}+1} \mathrm{~d} x, \\
d_{z_{0}}(u) & :=\frac{1}{\sqrt{1+\left|\nabla \varphi_{x_{0}}\right|^{2}}}\left[\frac{\left|\partial_{t} u A^{\frac{1}{2}} \nabla \varphi_{x_{0}}-A^{\frac{1}{2}} \nabla u\right|^{2}}{2}+\frac{|u|^{p_{c}+1}}{p_{c}+1}\right], \\
\operatorname{Flux}\left(u, M_{S}^{T}\left(z_{0}\right)\right) & :=\int_{M_{S}^{T}\left(z_{0}\right)} d_{z_{0}}(u) \mathrm{d} \sigma .
\end{aligned}
$$

Dans la suite, la lettre $C$ désignera une constante qui peut changer d'une ligne à l'autre.

\section{Existence globale de solutions classiques}

Dans ce paragraphe, nous nous proposons de prouver le théorème 1.1. La démonstration se fait en plusieurs étapes. Pour simplifier, toutes les preuves de ce paragraphe sont données en dimension $d=3$.

Soient $\left.d \geq 3, p_{c}=(d+2) /(d-2), z_{0}=\left(t_{0}, x_{0}\right) \in\right] 0,+\infty\left[\times \mathbb{R}^{d}\right.$ et $u \in$ $\mathcal{C}^{2}\left(K\left(z_{0}\right) \backslash\left\{z_{0}\right\}\right)$ une solution classique de $(1)$.

\subsection{Estimation de l'énergie}

Lemme 3.1. - Pour tous réels a et $b$ tels que $a<b<t_{0}$, on $a$

$$
E\left(u, D\left(b, z_{0}\right)\right)+\operatorname{Flux}\left(u, M_{a}^{b}\left(z_{0}\right)\right)=E\left(u, D\left(a, z_{0}\right)\right) .
$$

Démonstration du lemme 3.1. - Nous multiplions l'équation (1) par $\partial_{t} u$, nous obtenons $0=\partial_{t} e(u)-\operatorname{div}_{x}\left(\partial_{t} u \cdot A \nabla_{x} u\right)$, puis nous intégrons sur le cône tronqué $K_{a}^{b}\left(z_{0}\right)$. Le résultat est alors une conséquence de la formule de Stokes. 
Remarque 3.1. - 1) La quantité $E\left(u, D\left(a, z_{0}\right)\right)$ est décroissante en $a$. Comme elle est positive, sa limite en $t_{0}$ existe et $\lim _{a \rightarrow t_{0}} \operatorname{Flux}\left(u, M_{a}^{t_{0}}\left(z_{0}\right)\right)=0$.

2) D'après l'inégalité de Hölder et (15), nous pouvons voir que si $\alpha<2$ et $\beta<p_{c}+1$, on a

$$
\lim _{a \rightarrow t_{0}} \int_{D\left(a, z_{0}\right)}\left(\left|\partial_{t} u\right|^{\alpha}+\left|A^{\frac{1}{2}} \nabla_{x} u\right|^{\alpha}\right) \mathrm{d} x=\lim _{a \rightarrow t_{0}} \int_{D\left(a, z_{0}\right)}|u|^{\beta} \mathrm{d} x=0
$$

et la question naturelle que nous posons est : peut on étendre ces résultats pour $\alpha=2$ ou $\beta=p_{c}+1$, c'est-à-dire lorsqu'il s'agit de termes d'énergie ? La réponse est dans ce qui suit.

\subsection{Le lemme fondamental}

Lemme 3.2. - Pour tous réels a et $b$ tels que $a<b<t_{0}$, on $a$

$$
\begin{aligned}
& \int_{D\left(a, z_{0}\right)}|u(a, x)|^{p_{c}+1} \mathrm{~d} x \\
& \leq C_{0}(A)\left\{\begin{aligned}
& \frac{t_{0}-b}{t_{0}-a}\left(E\left(u, D\left(a, z_{0}\right)\right)+E^{(d-2) / d}\left(u, D\left(a, z_{0}\right)\right)\right) \\
&+ E\left(u, D\left(a, z_{0}\right)\right)-E\left(u, D\left(b, z_{0}\right)\right) \\
&+ {\left[E\left(u, D\left(a, z_{0}\right)\right)-E\left(u, D\left(b, z_{0}\right)\right)\right]^{(d-2) / d} } \\
&\left.+(b-a)\left(E\left(u, D\left(a, z_{0}\right)\right)+E^{(d-2) / d}\left(u, D\left(a, z_{0}\right)\right)\right)\right\}
\end{aligned}\right.
\end{aligned}
$$

où $C_{0}(A)$ est une constante dépendant de la métrique $A$.

Démonstration du lemme 3.2. - Nous prenons $z_{0}=(0,0)$ et nous notons par $\varphi$ la fonction $\varphi_{0}$, par $D(t)$ la boule $D(t,(0,0))$ et par $E(t)$ l'énergie locale $E(u, D(t))$. Quitte à renverser le temps et conserver les mêmes notations, nous pourrons travailler dans le cône d'avenir. Remarquons que l'inversion du temps conduit à estimer l'intégrale sur $D(b)$. Nous multiplions l'équation (1) par $L_{\varphi} u$ où

$$
L_{\varphi}=t \partial_{t}+\varphi A \nabla \varphi \cdot \nabla+1
$$

est tangentiel au cône géodésique issue du point $(0,0)$. Nous obtenons alors

$$
0=\partial_{t} q+\operatorname{div}_{x} P+R
$$

TOME $131-2003-\mathrm{N}^{\mathrm{O}} 1$ 
où l'on a posé

$$
\begin{aligned}
q(t, x):= & t\left[\frac{1}{2}\left|\frac{L_{\varphi} u}{t}\right|^{2}+\frac{A \nabla u \cdot \nabla u-\left(\varphi^{2} / t^{2}\right)(A \nabla \varphi \nabla u)^{2}}{2}+\frac{u^{6}}{6}\right]+\frac{u^{2}}{t}, \\
P(t, x):= & {\left[-\frac{\left(\partial_{t} u\right)^{2}}{2}+\frac{A \nabla u \cdot \nabla u+u^{2} / t^{2}-2 \partial_{t} u \cdot(u / t)}{2}+\frac{u^{6}}{6}\right] \varphi A \nabla \varphi-L_{\varphi} u A \nabla u, } \\
R(t, x):= & \left(3-\operatorname{div}_{x}(\varphi A \nabla \varphi)\right)\left[\frac{A \nabla u \cdot \nabla u}{2}+\frac{u^{6}}{6}+\frac{u^{2}}{2 t^{2}}-\frac{\left(\partial_{t} u\right)^{2}}{2}-\partial_{t} u \frac{u}{t}\right] \\
& +B(A \nabla u) \cdot \nabla u+\varphi \sum_{i, j, k, \ell} a_{k \ell} \partial_{k}\left(a_{i j}\right) \partial_{i} u\left(\partial_{j} \varphi \partial_{\ell} u-\partial_{\ell} \varphi \frac{\partial_{j} u}{2}\right)+\frac{u^{6}}{3} \\
= & I+I I+I I I+I V .
\end{aligned}
$$

La fonction matricielle $B$ du terme $I I$ est celle donnée par (14).

En intégrant (24) sur le cône d'avenir tronqué (encore noté $K_{a}^{b}$ ) et en utilisant les résultats de la proposition 2.1, nous obtenons

(25) $Q(b)-Q(a)+\int_{M_{a}^{b}}(-q+\nabla \varphi \cdot P) \frac{\mathrm{d} \sigma}{\sqrt{1+|\nabla \varphi|^{2}}}+\int_{K_{a}^{b}} R \mathrm{~d} x \mathrm{~d} t=0$,

avec $Q(s)=\int_{D(s)} q(s, x) \mathrm{d} x$. Par ailleurs sur le manteau $M_{a}^{b}$ nous avons, en vertu de la proposition $2.1, q-\nabla \varphi \cdot P=t\left|L_{\varphi} u / t\right|^{2}$ ce qui permet de réécrire (25)

$$
Q(b)-Q(a)-\int_{M_{a}^{b}} t\left|\frac{L_{\varphi} u}{t}\right|^{2} \frac{\mathrm{d} \sigma}{\sqrt{1+|\nabla \varphi|^{2}}}+\int_{K_{a}^{b}} R \mathrm{~d} x \mathrm{~d} t=0 .
$$

D'autre part, en utilisant (12) et l'inégalité de Hölder nous obtenons

$$
\int_{D(t)}|u(t, x)|^{6} \mathrm{~d} x \leq C \frac{1}{t} Q(t) \leq C\left(E(t)+E^{1 / 3}(t)\right),
$$

et

$$
\begin{aligned}
\int_{M_{a}^{b}} t \mid & \left.\frac{L_{\varphi} u}{t}\right|^{2} \frac{\mathrm{d} \sigma}{\sqrt{1+|\nabla \varphi|^{2}}} \\
& \leq C \int_{M_{a}^{b}}\left[b \cdot\left|\partial_{t} u A^{1 / 2} \nabla \varphi+A^{1 / 2} \nabla u\right|^{2}+\frac{u^{2}}{t}\right] \frac{\mathrm{d} \sigma}{\sqrt{1+|\nabla \varphi|^{2}}} \\
& \leq C b\left[E(b)-E(a)+(E(b)-E(a))^{1 / 3}\right]
\end{aligned}
$$

La dernière inégalité est une conséquence de l'inégalité de Hölder et de la propriété (15).

Il reste à estimer le reste $R$. En utilisant (13), (14) et (15) nous avons

$$
\int_{K_{a}^{b}}|I| \mathrm{d} x \mathrm{~d} t \leq C b(b-a)\left(E(b)+E(b)^{1 / 3}\right)
$$

et

$$
\int_{K_{a}^{b}}\{|I I|+|I I I|\} \mathrm{d} x \mathrm{~d} t \leq C b(b-a) E(b),
$$

BULLETIN DE LA SOCIÉTÉ MATHÉMATIQUE DE FRANCE 
ce qui, en vertu du signe de $I V$, achève la preuve du lemme 3.2.

Corollaire 3.1. - On a

$$
\lim _{a \rightarrow t_{0}} \int_{D(a)}|u(a, x)|^{p_{c}+1} \mathrm{~d} x=0 .
$$

Démonstration du corollaire 3.1. - Dans l'inégalité du lemme 3.2, nous choisissons $b=t_{0}-\varepsilon\left(t_{0}-a\right)$. En passant à la limite supérieure quand $a$ tend vers $t_{0}$, nous obtenons : pour tout $0<\varepsilon<1$,

$$
\varlimsup_{a \rightarrow t_{0}} \int_{D(a)}|u(a, x)|^{6} \mathrm{~d} x \leq C \varepsilon
$$

et par suite le résultat.

Remarque 3.2. - Le corollaire précédent montre que la partie non linéaire de l'énergie ne présente pas d'effet de concentration. Nous montrons par la suite qu'en fait cela est vrai pour toute l'énergie.

Corollaire 3.2. - Pour tout couple de Strichartz $(q, r)$, on a

$$
u \in L^{q}\left(L^{r}\left(K_{0}^{t_{0}}\right)\right) .
$$

Démonstration du corollaire 3.2. - Nous utilisons une version localisée des inégalités (6) dans des cônes tronqués $K_{s}^{t}\left(z_{0}\right)$ avec $0 \leq s \leq t<t_{0}$. En effet toute fonction $v=v(t, x)$ satisfait (voir proposition 4.4 de l'appendice)

$\|v\|_{L^{q}\left(L^{r}\left(K_{s}^{t}\right)\right)} \leq c_{q}\left[E_{0}^{\frac{1}{2}}\left(v, D\left(s, z_{0}\right)\right)+\|v\|_{L^{6}\left(D\left(s, z_{0}\right)\right)}+\int_{s}^{t}\left\|\square_{A} v\right\|_{L^{2}\left(D\left(\tau, z_{0}\right)\right)} \mathrm{d} \tau\right]$,

où $(q, r)$ est un couple admissible de Strichartz. En considérant le terme $|u|^{4} u$ comme un terme source dans l'inégalité précédente, nous avons

$$
\begin{aligned}
& \|u\|_{L^{q}\left(L^{r}\left(K_{s}^{t}\left(z_{0}\right)\right)\right)} \\
& \quad \leq c_{q}\left[E_{0}^{\frac{1}{2}}\left(u, D\left(s, z_{0}\right)\right)+\|u\|_{L^{6}\left(D\left(s, z_{0}\right)\right)}+\|u\|_{L^{5}\left(L^{10}\left(K_{s}^{t}\left(z_{0}\right)\right)\right.}^{5}\right] .
\end{aligned}
$$

En choisissant $(q, r)=(4,12)$ dans $(27)$ et en remarquant que l'énergie $E\left(u, D\left(s, z_{0}\right)\right)$ est majorée par l'énergie initiale $E_{0}$, nous obtenons

$$
\|u\|_{L^{4}\left(L^{12}\left(K_{s}^{t}\right)\right)} \leq c\left[E_{0}^{\frac{1}{2}}+\sup _{s \leq \tau \leq t}\|u\|_{L^{6}\left(D\left(\tau, z_{0}\right)\right)} \cdot\|u\|_{L^{4}\left(L^{12}\left(K_{s}^{t}\right)\right)}^{4}\right] .
$$

Ceci entraîne que pour tout $\varepsilon>0$, pour $s$ assez proche de $t_{0}$ et $s \leq t<t_{0}$, nous avons

$$
\|u\|_{L^{4}\left(L^{12}\left(K_{s}^{t}\left(z_{0}\right)\right)\right)} \leq c\left[E_{0}^{\frac{1}{2}}+\varepsilon\|u\|_{L^{4}\left(L^{12}\left(K_{s}^{t}\right)\right)}^{4}\right] .
$$

Pour $\varepsilon<\varepsilon_{0}:=2^{-6} c^{-5} E_{0}^{-2}$, le lemme 1.3 montre que $u \in L^{4}\left(L^{12}\left(K_{s}^{t}\left(z_{0}\right)\right)\right)$. Par interpolation des cas $(\infty, 6)$ et $(4,12)$, nous déduisons que $u \in L^{q}\left(L^{r}\left(K_{s}^{t}\left(z_{0}\right)\right)\right)$ pour tout $t<t_{0}$, et par suite le corollaire.

TOME $131-2003-\mathrm{N}^{\mathrm{O}} 1$ 
Corollaire 3.3. - On a

$$
\lim _{s \rightarrow t_{0}} E\left(u, D\left(s, z_{0}\right)\right)=0 .
$$

Démonstration du corollaire 3.3. - Pour $s<t_{0}$ assez proche de $t_{0}$, soit $v_{s}$ la solution de $\square_{A} v_{s}=0$ avec les données de Cauchy $v_{s}(s)=u(s)$ et $\partial_{t} v_{s}(s)=$ $\partial_{t} u(s)$. Alors la différence $w_{s}=u-v_{s}$ satisfait

$$
\square_{A} w_{s}=-u^{5}, \quad w_{s}(s)=\partial_{t} w_{s}(s)=0 .
$$

Nous en déduisons que $E_{0}^{\frac{1}{2}}\left(w_{s}, D\left(t, z_{0}\right)\right) \leq C\|u\|_{L^{5}\left(L^{10}\left(K_{s}^{t_{0}}\left(z_{0}\right)\right)\right)}^{5}$ pour $s<t<t_{0}$, où l'on a posé

$$
E_{0}\left(w_{s}, D\left(t, z_{0}\right)\right)=\frac{1}{2} \int_{D\left(t, z_{0}\right)}\left[\left(\partial_{t} w_{s}\right)^{2}+\left|\nabla w_{s}\right|^{2}\right](t, x) \mathrm{d} x .
$$

De l'inégalité triangulaire, il vient

$$
E_{0}^{\frac{1}{2}}\left(u, D\left(t, z_{0}\right)\right) \leq E_{0}^{\frac{1}{2}}\left(v_{s}, D\left(t, z_{0}\right)\right)+C\|u\|_{L^{5}\left(L^{10}\left(K_{s}^{t}\left(z_{0}\right)\right)\right)}^{5} .
$$

Par ailleurs, en utilisant le corollaire précédent et le fait que $E_{0}\left(v_{s}, D\left(t, z_{0}\right)\right)$ tend vers 0 quand $t \rightarrow t_{0}$, nous achevons la preuve de ce résultat.

Corollaire 3.4. - Supposons que $3 \leq d<6$. Alors il existe $s_{0}<t_{0}$ tel que

$$
u \in L^{4 /(d-2)}\left(\left[s_{0}, t_{0}\left[; L^{4 d /(d-2)}\left(\mathbb{R}^{d}\right)\right) .\right.\right.
$$

Démonstration du corollaire 3.4. - Pour $R>0$ et $S<T$, nous désignons par

$$
\Gamma_{S}^{T}(R)=\{z=(t, x), S \leq t \leq T \text { et }|x| \geq R+t\} .
$$

Pour la preuve du corollaire 3.4, nous vérifions d'abord que $u \in L^{4}\left(L^{12}\right)\left(\Gamma_{0}^{t_{0}}\left(R_{0}\right)\right)$. Ensuite, nous prouvons que l'énergie locale reste petite sur les sections des cônes tronqués issus des points $(t, x)$ avec $t>t_{0}$. En combinant ce résultat avec les inégalité de Strichartz nous démontrons que

$$
u \in L^{4}\left(L^{12}\left(R_{0}+s_{0} \leq|x| \leq R_{0}+t_{0}\right)\right)
$$

pour un certain $s_{0}>0$ convenablement choisi.

Notons par $u_{c}$ la solution de Shatah-Struwe du cas constant avec les mêmes données de Cauchy $\left(u_{0}, u_{1}\right)$. Par unicité, nous avons $u=u_{c}$ dans $\Gamma_{0}^{t}\left(R_{0}\right)$ pour tout $0 \leq t<t_{0}$. De plus, $u_{c} \in L^{q}\left(\mathbb{R}, L^{r}\left(\mathbb{R}^{3}\right)\right.$ ) (voir [2]) pour tout couple de Strichartz $(q, r)$. Il en résulte alors que $u \in L^{4}\left(L^{12}\right)\left(\Gamma_{0}^{t_{0}}\left(R_{0}\right)\right)$.

Soit $\varepsilon_{0}>0$ fixé. Pour tout $y \in \mathbb{R}^{3}$, il existe $\eta=\eta\left(\varepsilon_{0}, y\right)$ tel que :

$$
\left(0<t_{0}-t<\frac{1}{2} \eta\right) \text { implique }\left(\int_{\varphi_{y}(x) \leq t_{0}-t} e(u)\left(t_{0}-\frac{1}{2} \eta, x\right) \mathrm{d} x \leq \varepsilon_{0}\right) .
$$

BULLETIN DE LA SOCIÉTÉ MATHÉMATIQUE DE FRANCE 
De la continuité de l'application $t \mapsto \int_{\varphi_{y}(x) \leq t_{0}-t} e(u)\left(t_{0}-\frac{1}{2} \eta, x\right) \mathrm{d} x$ au point $t_{0}-\frac{1}{2} \eta$, il existe $\delta=\delta\left(\varepsilon_{0}, y\right)$ tel que

$$
\int_{\varphi_{y}(x) \leq \frac{1}{2} \eta+\delta} e(u)\left(t_{0}-\frac{1}{2} \eta, x\right) \mathrm{d} x \leq \varepsilon_{0} .
$$

En utilisant la décroissance de l'énergie (lemme 3.1), nous obtenons

$$
\sup _{t_{0}-\frac{1}{2} \eta \leq t<t_{0}} \int_{\varphi_{y}(x) \leq t_{0}+\delta-t} e(u)(t, x) \mathrm{d} x \leq \varepsilon_{0} .
$$

Notons par $K=\left\{(t, x),|x| \leq R_{0}+t_{0}\right.$ et $\left.0 \leq t \leq t_{0}\right\}$. Par un argument de compacité, nous pouvons écrire que

$$
K \subset \bigcup_{1 \leq j \leq n\left(\varepsilon_{0}\right)} K_{0}^{t_{0}}\left(\bar{z}_{j}\right)
$$

avec $\bar{z}_{j}=\left(t_{0}+\delta_{j}, y_{j}\right)$. Soit $s_{0}=\max _{1 \leq j \leq n\left(\varepsilon_{0}\right)}\left(t_{0}-\frac{1}{2} \eta_{j}\right)$. Écrivons les inégalités de Strichartz dans $K_{s_{0}}^{t_{0}}\left(\bar{z}_{j}\right)$; il vient que

$\|u\|_{L^{4}\left(L^{12}\left(K_{s_{0}}^{t_{0}}\left(\bar{z}_{j}\right)\right)\right.} \leq C\left[E^{\frac{1}{2}}\left(u, D\left(s_{0}, \bar{z}_{j}\right)+\|u\|_{L^{4}\left(L^{12}\left(K_{s_{0}}^{t_{0}}\left(\bar{z}_{j}\right)\right)\right.}^{4}\|u\|_{L^{\infty}\left(L^{6}\left(K_{s_{0}}^{t_{0}}\left(\bar{z}_{j}\right)\right)\right.}\right]\right.$.

Le lemme d'absorption 1.3 montre que pour tout $1 \leq j \leq n\left(\varepsilon_{0}\right)$, la solution $u \in L^{4}\left(L^{12}\left(K_{s_{0}}^{t_{0}}\left(\bar{z}_{j}\right)\right)\right.$, et par suite $u \in L^{4}\left(L^{12}\left(R_{0}+s_{0} \leq|x| \leq R_{0}+t_{0}\right)\right)$. Ce qui achève la preuve du corollaire.

Corollaire 3.5. - Il existe un réel $s_{1}, s_{0} \leq s_{1}<t_{0}$, tel que :

$$
\begin{array}{ll}
\langle D\rangle^{1} u \in L^{\infty}\left(\left[s_{1}, t_{0}\left[, L^{p_{c}+1}\left(\mathbb{R}^{d}\right)\right)\right.\right. & \text { pour } d \geq 3, \\
\langle D\rangle^{2} u \in L^{\infty}\left(\left[s_{1}, t_{0}\left[, L^{p_{c}+1}\left(\mathbb{R}^{d}\right)\right)\right.\right. & \text { pour } 3 \leq d \leq 6 .
\end{array}
$$

$\langle D\rangle^{\sigma}$ désigne le multiplicateur de Fourier associé à la fonction $\left(1+|\xi|^{2}\right)^{\frac{1}{2} \sigma}$.

Démonstration du corollaire 3.5. - Remarquons que $\langle D\rangle^{\sigma} u$ est solution de

$$
\square_{A}\left(\langle D\rangle^{\sigma} u\right)+R_{1, \sigma}\langle D\rangle^{\sigma} u+\langle D\rangle^{\sigma} u^{5}=0 .
$$

$R_{1, \sigma}$ est un opérateur pseudo-différentiel d'ordre 1. En appliquant les inégalités (6), nous obtenons

$$
\text { (28) } \begin{aligned}
\left\|\langle D\rangle^{1} u\right\|_{L^{q}\left(\left[s, t_{0}\left[, L^{r}\left(\mathbb{R}^{3}\right)\right)\right.\right.} & \leq C\left[\left(E_{0}^{\frac{1}{2}}\left(\langle D\rangle^{1} u, s\right)+\left\|\langle D\rangle^{1} u^{5}\right\|_{L^{1}\left(\left[s, t_{0}\left[, L^{2}\left(\mathbb{R}^{3}\right)\right)\right.\right.}\right]\right. \\
& \leq C\left[\left(E_{0}^{\frac{1}{2}}\left(\langle D\rangle^{1} u, 0\right)+\left\|\langle D\rangle^{1} u^{5}\right\|_{L^{1}\left(\left[s, t_{0}\left[, L^{2}\left(\mathbb{R}^{3}\right)\right)\right.\right.}\right] .\right.
\end{aligned}
$$

D'après la formule de Plancherel nous pouvons écrire

$$
\left\|\langle D\rangle^{1} u^{5}\right\|_{L^{2}\left(\mathbb{R}^{3}\right)}^{2}=\left\|u^{5}\right\|_{L^{2}\left(\mathbb{R}^{3}\right)}^{2}+C \int_{\mathbb{R}^{3}} \sum_{j=1}^{3}\left|\partial_{j} u^{5}\right|^{2}(x) \mathrm{d} x .
$$

TOME $131-2003-\mathrm{N}^{\mathrm{O}} 1$ 
En appliquant l'inégalité de Hölder à chacun des termes nous obtenons

$$
\begin{aligned}
\left\|\langle D\rangle^{1} u^{5}\right\|_{L^{2}\left(\mathbb{R}^{3}\right)}^{2} & \leq C\|u\|_{L^{12}\left(\mathbb{R}^{3}\right)}^{8}\left(\|u\|_{L^{6}\left(\mathbb{R}^{3}\right)}^{2}+\sum_{j=1}^{3}\left\|\partial_{j} u\right\|_{L^{6}\left(\mathbb{R}^{3}\right)}^{2}\right) \\
& \leq C\|u\|_{L^{12}\left(\mathbb{R}^{3}\right)}^{8} \cdot\left\|\langle D\rangle^{1} u\right\|_{L^{6}\left(\mathbb{R}^{3}\right)}^{2} .
\end{aligned}
$$

Et par suite

$$
\left\|\langle D\rangle^{1} u^{5}\right\|_{L^{1}\left(\left[s, t_{0}\left[, L^{2}\left(\mathbb{R}^{3}\right)\right)\right.\right.} \leq C\|u\|_{L^{4}\left(\left[s, t_{0}\left[, L^{12}\left(\mathbb{R}^{3}\right)\right)\right.\right.}^{4} \cdot\left\|\langle D\rangle^{1} u\right\|_{L^{\infty}\left(\left[s, t_{0}\left[, L^{6}\left(\mathbb{R}^{3}\right)\right)\right.\right.} .
$$

Pour le choix $(q, r)=(\infty, 6)$ dans $(28)$ et pour $s$ assez proche de $t_{0}$, nous pouvons rendre la quantité $\|u\|_{L^{4}\left(\left[s, t_{0}\left[, L^{12}\left(\mathbb{R}^{3}\right)\right)\right.\right.}$ aussi petite que l'on veut. Ce qui prouve que $\langle D\rangle^{1} u$ appartient à $L^{\infty}\left(\left[s_{1}, t_{0}\left[, L^{6}\left(\mathbb{R}^{3}\right)\right)\right.\right.$ pour $s_{1}$ convenablement fixé. En réappliquant les inégalités $(28)$, nous déduisons que $\langle D\rangle^{1} u$ appartient à $L^{q}\left(\left[s_{1}, t_{0}\left[, L^{r}\left(\mathbb{R}^{d}\right)\right)\right.\right.$, pour tout couple de Strichartz $(q, r)$.

D'autre part, nous écrivons les inégalités (28) pour $v:=\langle D\rangle^{2} u$. Nous obtenons

$$
\begin{aligned}
& \|v\|_{L^{q}\left(\left[s, t_{0}\left[, L^{r}\left(\mathbb{R}^{d}\right)\right)\right.\right.} \\
& \leq C \\
& C\left[\left(E_{0}^{\frac{1}{2}}(v, 0)+\|v\|_{L^{\infty}\left(\left[s, t_{0}\left[, L^{2 d /(d-2)}\left(\mathbb{R}^{d}\right)\right)\right.\right.} \cdot\|u\|_{L^{4 /(d-2)}\left(\left[s, t_{0}\left[, L^{4 d /(d-2)}\left(\mathbb{R}^{d}\right)\right)\right.\right.}\right.\right. \\
& \left.\quad+\left\|\langle D\rangle^{1} u\right\|_{L^{2 d /(d-2)}\left(\left[s, t_{0}\left[, L^{4 d /(d-2)}\left(\mathbb{R}^{d}\right)\right)\right.\right.}^{2} \cdot\|u\|_{L^{2 d /(d-2)\left(\left[s, t_{0}\left[, L^{4 d /(d-2)}\left(\mathbb{R}^{d}\right)\right)\right.\right.}}^{(6-d) /(d-2)}\right]
\end{aligned}
$$

ce qui justifie la restriction sur la dimension et achève la preuve.

Fin de la démonstration du théorème 1.1. - Nous utilisons le résultat suivant (voir, par exemple, [14]).

LEMME 3.3. - Le problème (3) admet une unique solution classique maximale $u$ définie sur $]-T^{*}, T^{*}\left[\times \mathbb{R}^{d}\right.$ avec l'une de deux alternatives suivantes :

(i) $T^{*}=+\infty$,

(ii) $T^{*}<+\infty$ et $\varlimsup_{t \rightarrow T^{*}}\|u(t, .)\|_{L^{\infty}\left(\mathbb{R}^{d}\right)}=+\infty$.

Supposons que $T^{*}<+\infty$, alors $\varlimsup_{t \rightarrow T^{*}}\|u(t)\|_{L^{\infty}}=+\infty$. Or d'après les corollaires 3.4 et 3.6 , nous avons $u \in L^{\infty}\left(\left[s_{1}, T^{*}\left[, W^{1,6}\left(\mathbb{R}^{3}\right)\right)\right.\right.$ où $W^{1,6}$ désigne l'espace de Sobolev usuel. Ce qui entraîne le résultat en utilisant l'injection de Sobolev $\left.W^{1,6}\left(\mathbb{R}^{3}\right)\right) \hookrightarrow L^{\infty}\left(\mathbb{R}^{3}\right)$.

Pour les dimensions $4 \leq d<6$, nous devons « dériver » l'équation (1) deux fois et utiliser l'injection de Sobolev

$$
W^{2,2 d /(d-2)}\left(\mathbb{R}^{d}\right) \hookrightarrow L^{\infty}\left(\mathbb{R}^{d}\right), \quad 4 \leq d<6 .
$$

BULLETIN DE LA SOCIÉtÉ MATHÉMATIQUE DE FRANCE 


\section{Existence globale dans l'espace d'énergie}

Nous discutons maintenant l'existence globale et l'unicité pour des données de Cauchy dans l'espace d'énergie $\mathcal{E}=\dot{H}^{1} \times L^{2}$. Nous avons

ThÉorème 4.1. - Soient $\left(u_{0}, u_{1}\right) \in \mathcal{E}, d \geq 3$ et $p_{c}=(d+2) /(d-2)$. Alors le problème

$$
\square_{A} u+|u|^{p_{c}-1} u=0, \quad\left(u, \partial_{t} u\right)(0, .)=\left(u_{0}, u_{1}\right),
$$

admet une unique solution $u$ telle que

$$
\left(u, \partial_{t} u\right) \in\left\{\mathcal{C}\left(\mathbb{R}, \dot{H}^{1}\right) \cap L_{\text {loc }}^{p_{c}}\left(\mathbb{R}, L^{2 p_{c}}\right)\right\} \times \mathcal{C}\left(\mathbb{R}, L^{2}\right) .
$$

Avant de prouver ce résultat, précisons d'abord quelques notations.

4.1. Notations. - (a) Nous noterons par $v$ la solution de l'équation libre avec les mêmes données de Cauchy

$$
\square_{A} v=0, \quad\left(v, \partial_{t} v\right)(0, .)=\left(u_{0}, u_{1}\right) .
$$

(b) Soit $\delta_{0}>0$ défini par $p_{c} c 2^{p_{c}-1} \delta_{0}^{p_{c}-1} \leq \frac{1}{4}$ avec $c=\max \left(c(0), c_{p_{c}}\right)$ où les constantes $c(0)$ et $c_{p_{c}}$ sont données respectivement par (5) et (6).

(c) Nous notons par $T_{0}$ un réel positif tel que $\|v\|_{L^{p_{c}}\left(\left[0, T_{0}\right], L^{2 p_{c}}\right)} \leq \delta_{0}$.

(d) Pour tout $I$, intervalle de temps compact, $\mathcal{E}(I)$ désigne l'espace

$$
\mathcal{E}(I):=\left\{u \in \mathcal{C}\left(I, \dot{H}^{1}\right) \cap \mathcal{C}^{1}\left(I, L^{2}\right) \text { et telle que } u \in L^{p_{c}}\left(I, L^{2 p_{c}}\right)\right\} .
$$

(e) Pour tout $T>0, \mathcal{F}_{T}$ désigne le sous espace de $\mathcal{E}([0, T])$ formé des éléments $w$ vérifiant de plus $w(0,)=.\partial_{t} w(0,)=$.0 .

Remarque 4.1. - 1) Nous posons

$$
\|u\|_{\mathcal{E}(I)}=\sup _{I}\left(\|u(t, .)\|_{\dot{H}^{1}}+\left\|\partial_{t} u(t, .)\right\|_{L^{2}}\right)+\|u\|_{L^{p_{c}\left(I, L^{2 p_{c}}\right)}} .
$$

Muni de la norme $\|\cdot\|_{\mathcal{E}(I)}$, l'espace $\mathcal{E}(I)$ est un sous-espace de $L^{\infty}(I, \mathcal{E})$ qui englobe toutes les propriétés d'intégrabilité espace-temps de la solution. Précisément, si $\|u\|_{\mathcal{E}(I)}<+\infty$, alors pour tout couple $(q, r)$ vérifiant $(\mathrm{S}),\|u\|_{L^{q}\left(I, L^{r}\right)}<$ $+\infty$.

2) $\mathcal{F}_{T_{0}}$ est un sous-espace fermé de $\mathcal{E}\left(\left[0, T_{0}\right]\right)$.

$3)$ Une solution de $(1)$ dans $\mathcal{E}([0, T])$ vérifie la loi de conservation de l'énergie

$$
E(u, t)=\int_{\mathbb{R}^{d}}\left\{\frac{1}{2}\left(\left(\partial_{t} u\right)^{2}+\left|A^{\frac{1}{2}} \nabla_{x} u\right|^{2}\right)+\frac{|u|^{p_{c}+1}}{p_{c}+1}\right\} \mathrm{d} x=E(u, 0) .
$$

4) Dans la suite de cette section, nous nous limiterons à la dimension $d=3$.

TOME $131-2003-\mathrm{N}^{\mathrm{O}} 1$ 


\subsection{Existence locale}

Proposition 4.1. - Soit $T_{0}$ donné par (c). Alors (30) admet une solution $u$ dans $\mathcal{E}\left(\left[0, T_{0}\right]\right)$.

Démonstration de la proposition 4.1. - La preuve est basée sur une application du théorème du point fixe dans $B_{\delta_{0}}$ : la boule fermée de centre 0 et de rayon $\delta_{0}$ de l'espace $\mathcal{F}_{T_{0}}$. Soit $\Phi$ l'application définie par

$$
\Phi: B_{\delta_{0}} \longrightarrow B_{\delta_{0}}, \quad w \longmapsto \tilde{w}
$$

solution de

$$
\square_{A} \tilde{w}=-(v+w)^{5}, \quad \tilde{w}(0, .)=\partial_{t} \tilde{w}(0, .)=0 .
$$

(i) $\Phi$ est bien définie : en appliquant les inégalités (5) et (6), nous obtenons

$$
\begin{gathered}
\|\tilde{w}\|_{L^{5}\left(\left[0, T_{0}\right], L^{10}\right)} \leq c\|v+w\|_{L^{5}\left(\left[0, T_{0}\right], L^{10}\right)}^{5} \leq \frac{\delta_{0}}{10} \\
\sup _{\left[0, T_{0}\right]}\left(\|\tilde{w}(t, .)\|_{\dot{H}^{1}}+\left\|\partial_{t} \tilde{w}(t, .)\right\|_{L^{2}}\right) \leq \frac{\delta_{0}}{10}
\end{gathered}
$$

(ii) $\Phi$ est contractante : en appliquant l'inégalité (6), nous avons

$$
\left\|\Phi\left(w_{1}\right)-\Phi\left(w_{2}\right)\right\|_{L^{5}\left(\left[0, T_{0}\right], L^{10}\right)} \leq c\left\|\left(v+w_{2}\right)^{5}-\left(v+w_{1}\right)^{5}\right\|_{L^{1}\left(\left[0, T_{0}\right], L^{2}\right)},
$$

et l'inégalité de Hölder appliquée en espace puis en temps donne

$$
\begin{aligned}
\left\|\Phi\left(w_{1}\right)-\Phi\left(w_{2}\right)\right\|_{L^{5}\left(\left[0, T_{0}\right], L^{10}\right)} & \leq c\left\|w_{1}-w_{2}\right\|_{L^{5}\left(\left[0, T_{0}\right], L^{10}\right)} \sum_{j=0}^{4} 2^{j} \delta_{0}^{j} 2^{4-j} \delta_{0}{ }^{4-j} \\
& \leq \frac{1}{4}\left\|w_{1}-w_{2}\right\|_{L^{5}\left(\left[0, T_{0}\right], L^{10}\right)} .
\end{aligned}
$$

L'autre partie de la norme se traite de la même façon et finalement nous obtenons

$$
\left\|\Phi\left(w_{1}\right)-\Phi\left(w_{2}\right)\right\|_{\mathcal{E}\left(\left[0, T_{0}\right]\right)} \leq \frac{1}{2}\left\|w_{1}-w_{2}\right\|_{\mathcal{E}\left(\left[0, T_{0}\right]\right)} .
$$

D'après le théorème du point fixe, il existe un unique $w \in B_{\delta_{0}}$ solution de

$$
\square_{A} w=-(v+w)^{5}, \quad w(0, .)=\partial_{t} w(0, .)=0,
$$

et en prenant $u=v+w$, nous obtenons une solution de (30).

Conclusion. - Nous avons pu résoudre le problème de Cauchy (30) sur un intervalle $\left[0, T_{0}\right]$ où $T_{0}$ est donné par $(\mathrm{c}), \delta_{0}$ étant une constante universelle. 


\subsection{Unicité}

Proposition 4.2. - Soit $T>0$ donné. Alors le problème (30) admet au plus une solution dans $\mathcal{E}([0, T])$.

Démonstration de la proposition 4.2. - Par un argument standard utilisant l'invariance de l'équation (1) par des translations en temps, il suffit de prouver l'unicité pour $T$ assez petit.

Soient $u$ et $v$ deux solutions du problème (30) dans $\mathcal{E}([0, T])$. Alors $u-v$ vérifie $\|u-v\|_{L^{5}\left([0, T], L^{10}\right)} \leq c\left\|u^{5}-v^{5}\right\|_{L^{1}\left([0, T], L^{2}\right)}$ en vertu de (6). Soit en utilisant l'inégalité de Hölder

$\|u-v\|_{L^{5}\left([0, T], L^{10}\right)} \leq c\|u-v\|_{L^{5}\left([0, T], L^{10}\right)} \sum_{j=0}^{4}\|u\|_{L^{5}\left([0, T], L^{10}\right)}^{4-j} \cdot\|v\|_{L^{5}\left([0, T], L^{10}\right)}^{j}$.

Si $T$ est assez petit tel que $c \sum_{j=0}^{4}\|u\|_{L^{5}\left([0, T], L^{10}\right)}^{4-j}\|v\|_{L^{5}\left([0, T], L^{10}\right)}^{j}<1$, nous obtenons $u=v \operatorname{sur}[0, T] \times \mathbb{R}^{3}$.

4.4. Prolongement de la solution locale. - Nous nous restreindrons aux temps positifs. D'après ce qui précède, le problème (30) admet une unique solution maximale $u$ définie sur $\left[0, T^{*}\left[\times \mathbb{R}^{3}\right.\right.$. Sur $u$ nous avons le résultat suivant.

Proposition 4.3. - La solution u appartient à l'espace $L^{p_{c}}\left(\left[0, T^{*}\left[, L^{2 p_{c}}\right)\right.\right.$.

Démonstration de la proposition 4.3. - Notons d'abord que par définition de $T^{*} ; u \in L_{\mathrm{loc}}^{q}\left(\left[0, T^{*}\left[, L^{r}\right)\right.\right.$ pour tout couple de Strichartz $(q, r)$. D'autre part, un argument de régularisation identique à celui de [1] ou [16], permet d'établir l'analogue du lemme 3.2 pour la solution $u$.

Ensuite, nous pouvons voir qu'on peut répéter les preuves des corollaires 3.4, 3.5 , et par suite avoir le résultat.

Pour prolonger la solution locale, nous allons montrer l'existence de $v_{s}$ solution de

$$
\square_{A} v_{s}=0, \quad v_{s}(s)=u(s), \quad \partial_{t} v_{s}(s)=\partial_{t} u(s),
$$

et qui vérifie pour un certain $\delta>0$,

$$
\left\|v_{s}\right\|_{L^{5}\left(\left[s, T^{*}+\delta\left[, L^{10}\right)\right.\right.} \leq \delta_{0},
$$

ce qui permettra de prolonger la solution $u$ sur $\left[s, T^{*}+\delta[\right.$ et d'aboutir à une contradiction.

Pour $s \leq t<T^{*}$, nous pouvons écrire $u=v_{s}+w_{s}$ où

$$
\square_{A} w_{s}=-u^{5}, \quad w_{s}(s)=\partial_{t} w_{s}(s)=0 .
$$

Nous avons donc

$$
\left\|v_{s}\right\|_{L^{5}\left([s, t], L^{10}\right)} \leq\|u\|_{L^{5}\left([s, t], L^{10}\right)}+c\|u\|_{L^{5}\left([s, t], L^{10}\right)}^{5} .
$$

TOME $131-2003-\mathrm{N}^{\mathrm{O}} 1$ 
Or d'après la proposition $4.3, \lim _{s \rightarrow T^{*}}\|u\|_{L^{5}\left(\left[s, T^{*}\left[L^{10}\right)\right.\right.}=0$. Donc pour $s$ assez proche de $T^{*}$, nous obtenons $\left\|v_{s}\right\|_{L^{5}\left(\left[s, T^{*}\left[, L^{10}\right)\right.\right.} \leq \frac{1}{2} \delta_{0}$.

La continuité de l'application $t \mapsto\left\|v_{s}\right\|_{L^{5}\left([s, t], L^{10}\right)}$ en $T^{*}$ montre qu'il existe $\delta>0$ tel que

$$
\left\|v_{s}\right\|_{L^{5}\left(\left[s, T^{*}+\delta\left[, L^{10}\right)\right.\right.} \leq \delta_{0} .
$$

Ainsi, nous pouvons prolonger $u$ au delà de $T^{*}$, ce qui contredit la maximalité de $u$.

\section{Appendice}

\section{Estimations de Strichartz localisées}

Proposition 4.4. - Soient $\left.z_{0}=\left(t_{0}, x_{0}\right) \in\right] 0,+\infty\left[\times \mathbb{R}^{d}, d \geq 3\right.$ et $f \in$ $L^{1}\left(\mathbb{R}, L^{2}\left(\mathbb{R}^{d}\right)\right)$. Il existe un réel $s_{0}<t_{0}$ tel que, pour tout $s_{0}<S \leq t_{0}$ et pour toute solution $v$ de

$$
\square_{A} v=f, \quad v(S, .)=v_{0} \in \dot{H}^{1}, \quad \partial_{t} v(S, .)=v_{1} \in L^{2},
$$

nous avons

$$
\begin{aligned}
\|v\|_{L^{q}\left(L^{r}\left(K_{S}^{t_{0}}\left(z_{0}\right)\right)\right)} \leq c_{q}\left[\left\|\nabla v_{0}\right\|_{L^{2}\left(D\left(S, z_{0}\right)\right)}+\left\|v_{1}\right\|_{L^{2}\left(D\left(S, z_{0}\right)\right)}\right. & \\
& \left.+\left\|v_{0}\right\|_{L^{6}\left(D\left(S, z_{0}\right)\right)}+\|f\|_{L^{1}\left(L^{2}\left(K_{S}^{t_{0}}\left(z_{0}\right)\right)\right)}\right] .
\end{aligned}
$$

Démonstration de la proposition 4.4. - Nous prenons $x_{0}=0$. Soient $\varepsilon_{0}>0$ et $U_{0}$ un voisinage de 0 dans $\mathbb{R}^{d}$ tels que

$$
\exp _{0}:\left\{\|y\|_{0}<\varepsilon_{0}\right\} \longrightarrow U_{0}
$$

soit un $C^{\infty}$ difféomorphisme. Désignons par $w$ la solution du problème de Cauchy

$$
\square_{A} w=\mathbf{1}_{K_{S}^{t_{0}}\left(z_{0}\right)} f, \quad w(S, .)=P\left(\mathbf{1}_{D\left(S, z_{0}\right)} v_{0}\right), \quad \partial_{t} w(S, .)=\mathbf{1}_{D\left(S, z_{0}\right)} v_{1},
$$

où $P$ est l'opérateur de prolongement de $H^{1}\left(D\left(S, z_{0}\right)\right)$ dans $\dot{H}^{1}\left(\mathbb{R}^{d}\right)$ défini par

$$
(P g)(x)= \begin{cases}g(x) & \text { si } x \in D\left(S, z_{0}\right), \\ \chi\left(\frac{t_{0}-S}{\left|\exp _{0}^{-1}(x)\right|_{0}}\right) g\left(\exp _{0}\left(\left(t_{0}-S\right)^{2} \frac{\exp _{0}^{-1}(x)}{\left|\exp _{0}^{-1}(x)\right|_{0}^{2}}\right)\right) & \text { si } x \in \mathcal{C}\left(S, S^{\prime}, z_{0}\right) \\ 0 & \text { sinon, }\end{cases}
$$

$\chi$ étant une fonction $C^{\infty}$ vérifiant $\chi(p)=0$ pour $p \leq \frac{1}{4}$ et $\chi(p)=1$ pour $p \geq \frac{1}{2} ; \mathcal{C}\left(S, S^{\prime}, z_{0}\right)$ est la couronne définie $\operatorname{par} \mathcal{C}\left(S, S^{\prime}, z_{0}\right):=D\left(S^{\prime}, z_{0}\right) \backslash D\left(S, z_{0}\right)$.

BULLETIN DE LA SOCIÉTÉ MATHÉMATIQUE DE FRANCE 
Le réel $S^{\prime}$ est choisi tel que $\left(t_{0}-S^{\prime}\right)=4\left(t_{0}-S\right)$. Alors, en appliquant les inégalités de Strichartz à $w$, nous obtenons

$$
\begin{aligned}
\|v\|_{L^{q}\left(L^{r}\left(K_{S}^{t_{0}}\left(z_{0}\right)\right)\right)}= & \|w\|_{L^{q}\left(L^{r}\left(K_{S}^{t_{0}}\left(z_{0}\right)\right)\right)} \\
\leq & c_{q}\left[\left\|\nabla\left(P\left(\mathbf{1}_{D\left(S, z_{0}\right)} v_{0}\right)\right)\right\|_{L^{2}\left(\mathbb{R}^{3}\right)}\right. \\
& \left.\quad+\left\|v_{1}\right\|_{L^{2}\left(D\left(S, z_{0}\right)\right)}+\|f\|_{L^{1}\left(L^{2}\left(K_{S}^{t_{0}}\left(z_{0}\right)\right)\right)}\right] .
\end{aligned}
$$

Signalons que nous avons utilisé le fait que $w=v$ dans $K_{S}^{t_{0}}$. En effet, il est clair que l'identité d'énergie locale du lemme 3.1 a un équivalent pour l'équation linéaire. Pour conclure il suffit de prouver que

$$
\left\|\nabla\left(P\left(\mathbf{1}_{D\left(S, z_{0}\right)}\right) g\right)\right\|_{L^{2}\left(\mathbb{R}^{3}\right)} \leq C\left[\|\nabla g\|_{L^{2}\left(D\left(S, z_{0}\right)\right)}+\|g\|_{L^{6}\left(D\left(S, z_{0}\right)\right)}\right] .
$$

D'abord, nous écrivons la différentielle de $P\left(\mathbf{1}_{D\left(S, z_{0}\right)} g\right)$ dans $\mathcal{C}\left(S, S^{\prime}, z_{0}\right)$ :

$$
D\left(P\left(\mathbf{1}_{D\left(S, z_{0}\right)} v_{0}\right)\right)(x):=(I)+(I I)
$$

avec

$$
\begin{aligned}
(I):=-\frac{|y|_{0}}{\left(t_{0}-S\right)^{3}} \chi^{\prime}\left(\frac{|y|_{0}}{t_{0}-S}\right) g\left(\exp _{0}(y)\right) y \cdot D \exp _{0}^{-1}(x), \\
(I I):=\left(t_{0}-S\right)^{2} \chi\left(\frac{|y|_{0}}{t_{0}-S}\right) D g\left(\exp _{0}(y)\right) D \exp _{0}(y) \\
\times D \Phi\left(\left(t_{0}-S\right)^{2} \frac{y}{|y|_{0}^{2}}\right) D \exp _{0}^{-1}(x), \\
y=\left(t_{0}-S\right)^{2} \frac{\exp _{0}^{-1}(x)}{\left|\exp _{0}^{-1}(x)\right|_{0}^{2}} \quad \text { et } \quad \Phi(z)=\frac{z}{|z|_{0}^{2}}
\end{aligned}
$$

Ensuite, nous estimons

$$
\begin{aligned}
\int_{\mathcal{C}\left(S, S^{\prime}, z_{0}\right)}|(I)|^{2} \mathrm{~d} x \leq C\left(t_{0}-S\right)^{2} & \int_{\mathcal{C}\left(S, S^{\prime}, z_{0}\right)}\left|\exp _{0}^{-1}(x)\right|_{0}^{-4} \\
& \times g^{2}\left(\exp _{0}\left(\left(t_{0}-S\right)^{2} \frac{\exp _{0}^{-1}(x)}{\left.\mid \exp _{0}^{-1}(x)\right)\left.\right|_{0} ^{2}}\right)\right) \mathrm{d} x,
\end{aligned}
$$

où l'on a utilisé le fait que $\sup _{z \in \bar{U}_{0}}\left\|D \exp _{0}^{-1}(z)\right\|<\infty$ (quitte à réduire l'ouvert $\left.U_{0}\right)$. Avec le changement de variable $z=\exp _{0}(y)$ et en notant par $t_{0}-S^{\prime \prime}=\frac{1}{4}\left(t_{0}-S\right)$, nous obtenons

$$
\begin{aligned}
\int_{\mathcal{C}\left(S, S^{\prime}, z_{0}\right)}|(I)|^{2} \mathrm{~d} x & \leq C \int_{\mathcal{C}\left(S^{\prime \prime}, S, z_{0}\right)} \frac{g^{2}(z)}{\varphi_{0}^{2}(z)} \mathrm{d} z \\
& \leq C\|g\|_{L^{6}\left(D\left(S, z_{0}\right)\right)}^{2}\left(\int_{\frac{1}{4}\left(t_{0}-S\right)}^{t_{0}-S} \frac{\mathrm{d} r}{r}\right)^{\frac{2}{3}} \\
& \leq C\|g\|_{L^{6}\left(D\left(S, z_{0}\right)\right)}^{2}(\ln 4)^{\frac{2}{3}} .
\end{aligned}
$$

TOME $131-2003-\mathrm{N}^{\mathrm{O}} 1$ 
Par ailleurs, nous avons

$$
\begin{aligned}
\int_{\mathcal{C}\left(S, S^{\prime}, z_{0}\right)}|(I I)|^{2} \mathrm{~d} x & \leq C\left(t_{0}-S\right)^{4} \int_{\mathcal{C}\left(S^{\prime \prime}, S, z_{0}\right)}|\nabla g(z)|^{2} \frac{|y|_{0}^{4}}{\left(t_{0}-S\right)^{8}} \mathrm{~d} x \\
& \leq C \int_{\mathcal{C}\left(S^{\prime \prime}, S, z_{0}\right)}|\nabla g(z)|^{2} \varphi_{0}^{4}(z)\left(t_{0}-S\right)^{-8} \varphi_{0}^{-6}(z)\left(t_{0}-S\right)^{6} \mathrm{~d} z \\
& \leq C \int_{\mathcal{C}\left(S^{\prime \prime}, S, z_{0}\right)}|\nabla g(z)|^{2} \frac{\left(t_{0}-S\right)^{2}}{\varphi_{0}^{2}(z)} \mathrm{d} z \\
& \leq C \int_{\mathcal{C}\left(S^{\prime \prime}, S, z_{0}\right)}|\nabla g(z)|^{2} \mathrm{~d} z
\end{aligned}
$$

ce qui achève la preuve de la proposition 4.4 .

\section{BIBLIOGRAPHIE}

[1] BAhouri (H.) \& GÉRARD (P.) - High frequency approximation of solutions to critical nonlinear wave equations, Amer. J. Math., t. 121 (1999), pp. 131-175.

[2] Bahouri (H.) \& Shatah (J.) - Decay estimates for the critical semilinear wave equation, Ann. Inst. Henri Poincaré, Anal. non-linéaire, t. 15 (1998), pp. 783-789.

[3] Gallot (S.), Hulin (D.) \& Lafontaine (J.) - Riemannian geometry, second éd., Springer-Verlag, 1993.

[4] Ginibre (J.) \& Velo (G.) - The global Cauchy problem for nonlinear Klein-Gordon equation, Math. Zeit., t. 189 (1985), pp. 487-505.

[5] - Generalized Strichartz inequalities for the wave equations, J. Funct. Anal., t. 133 (1995), pp. 50-68.

[6] Grillakis (M.) - Regularity and asymptotic behaviour of the wave equation with a critical nonlinearity, Ann. of Math., t. 132 (1990), pp. 485-509.

[7] _ Regularity for the wave equation with a critical nonlinearity, Comm. Pure Appl. Math., t. XLVI (1992), pp. 749-774.

[8] Ibrahim (S.) \& Majdoub (M.) - Existence globale de solutions pour l'équation des ondes semi-linéaire critique à coefficients variables, C. R. Acad. Sci. Paris Sér. I Math., t. 328 (1999), pp. 579-584.

[9] Kapitanski (L.V.) - Some generalizations of the Strichartz-Brenner inequality, Leningrad Math. J., t. 1 (1990), no. 3, pp. 693-726.

[10] The Cauchy problem for a semilinear wave equation, Zap. Nauchn. Sem. Leningrad Otdel. Math. Inst. Steklov (LOMI), t. 181-182 (1990), pp. 24-64 and 38-85.

[11] _ The Cauchy problem for a semilinear wave equation II, J. Soviet Math., Série I, t. 62 (1992), pp. 2746-2777.

BULletin DE LA SOCiÉtÉ MATHÉmATIQUE DE FRANCE 
[12] Global and unique weak solutions of nonlinear wave equations, Math. Research Letters, t. 1 (1994), no. 2, pp. 211-224.

[13] Lebeau (G.) - Optique non linéaire et ondes sur-critiques, in Sém. équations dérivées partielles, École Polytechnique, Palaiseau, 1999-2000.

[14] MAJdA (A.) - Compressible fluid flow and systems of conservation lows in several space variables, Appl. Math. Sci., vol. 53, Springer, 1984.

[15] Shatah (J.) \& Struwe (M.) - Regularity results for nonlinear wave equations, Ann. of Math., t. 138 (1993), no. 2, pp. 503-518.

[16] _ Well-posedness in the energy space for semilinear wave equation with critical growth, IMRN, t. 7 (1994), pp. 303-309.

[17] Sмiтh (H.) - Une paramétrix pour les équations des ondes à coefficients $\mathcal{C}^{1,1}$, Ann. Inst. Fourier, t. 48 (1998), no. 3, pp. 797-835.

[18] Struwe (M.) - Semilinear wave equations, Bull. Amer. Math. Soc., t. 26 (1992), pp. 53-85.

[19] ZuILY (C.) - Solutions en grand temps d'équations d'ondes non linéaire, in Sém. Bourbaki 1993/1994, Astérisque, vol. 227, Soc. Math. France, 1995, exposé $n^{\circ} 779$. 